\title{
Effects of Staggered Arrangement of Square Cylinders with Detached Flat Plat on Vorticity and Sound Generation
}

\author{
Bukhari Manshoor ${ }^{1}$, MohdAzahari Razali ${ }^{2}$, Izzuddin Zaman ${ }^{3}$, Hafiz Zafar Sharif ${ }^{4}$ \\ ${ }^{1}$ Faculty of Mechanical and Manufacturing Engineering, \\ UniversitiTun Hussein Onn Malaysia, MALAYSIA, bukhari@uthm.edu.my \\ ${ }^{2}$ Centre for Energy and Industrial Environment Studies, \\ UniversitiTun Hussein Onn Malaysia, MALAYSIA, azahari@uthm.edu.my \\ ${ }^{3}$ Acoustics, Noise and Vibrations Research Group, \\ UniversitiTun Hussein Onn Malaysia, MALAYSIA, izzuddin@uthm.edu.my \\ ${ }^{4}$ Higher College of Technology, Muscat, OMAN, zafar.sharif@hct.edu.om
}

\begin{abstract}
Effects of detached flat plat on sound generation and vorticity in flow through square cylinder in the staggered arrangement were examined numerically at Reynolds numbers 500 to 3000. The purpose of this study is to investigate the characteristics of vorticity, dynamic pressure and direction of total pressure propagation. Besides that, the acoustic power level was determined to look at the effect of staggered arrangement on the sound generation. The simulation conducted with three configurations of staggered arrangement, $90^{\circ}, 60^{\circ}$ and $30^{\circ}$ of two square cylinders with the detached plate. Computational Fluid Dynamic (CFD) with ANSYS Software is used to perform the simulation. The results showed that the vortex formation easily occurs at low Reynolds number. At high Reynolds number, the characteristic of the flow propagation shows similar patterns. Less entrainment of the flow occurred and the vortex is reattached together formed a streamline at high Reynolds number. The sound produced is measured by using the acoustic power level. The higher the Reynolds number, the higher the sound occupied at the vicinity of the cylinders. The maximum acoustic power level is $30.3 d B$ which occurred at the $60^{\circ}$ arrangement.
\end{abstract}

Key words: Acoustic power level, sound generation, staggered arrangement, total pressure, vortex formation.

\section{INTRODUCTION}

Fluid flow over a bluff body is a fundamental and important knowledge in fluid mechanics and had been studying thoroughly in the last few decades. The concern characteristic of flow and sound generation on the flow through a square cylinder is one of the concerns in the most design industry especially in aircraft and automotive industry. The generation of unwanted sounds due to the moving object that will degrade the performance of the vehicles [1] is one reason of the important thing on research on a flow induced-noise [2]. This flow-induced noise is one of the complex engineering problems that always associated with bluff bodies [3]. For the flow over the bluff body, vortex shedding occurred for which the fluid flow fluctuated on top and bottom of the bodies, creates the shear layer that separates the positive and negative pressure around the bodies. As the fluctuation of pressure continues to move downstream, the vortices will be formed and formation of these vortices will generate strong tonal noises that generate sound pressure [4]. The generation of this sound is due to the periodically surface fluctuation on the bluff bodies. The bluff body can have a simple geometry but the fluid flow over that particular bluff body has a relatively complex flow structure especially at the near wake. This phenomenon can be occurred for example in case of wind turbine [5].

Basically, the reduction of unwanted sound caused by the phenomena of flow-induced noise is one of the most fundamental and practically important problems in fluid dynamics and aeroacoustics. From Curle's acoustic analogy [6], a lift fluctuation exerted on the cylinder is responsible for the reduction of the sound pressure propagation. Active and passive methods have been used to transform the development vortex shedding behind the cylinder. This method is intended to suppress the aerodynamic force and/or flow due to it cylinder vibration. A rotational cylinder is one of the active control of vortex shedding [7], as well as velocity fluctuations in the wake using as a feedback control by a signal $[8,9]$.Another way that can be used to control the vortex shedding is by vibration which is the oscillation of the cylinder both normal to and in-line with the incident mean flow [10].Another method that was used to suppress the vortex shedding is by using a synthetic jet [11]and plasma actuators [12,13].For the passive method, detached plates have often been used. Roshko [14] was showed that the attachment of detached plate can reduced the drag of pressure significantly. Research by Apelt and West [15] showed that an 
implementation of detached plate more than 2Dcould progressively modify the drag and vortex shedding up to 5D. The detached plate longer than 5D shows no further change on the drag and vortex shedding.

In the engineering practical application, the behaviour of fluid flow over a bluff body in the various flow conditions need to be well understood. Sound generation due to the flow past the cylinders in various arrangements is one of the engineering problems associated with bluff bodies. The periodic surface-pressure fluctuations from vortex shedding create a strong tonal noise.

For that, the sound generation mechanism will be evaluated in term of the effect of different arrangement, differentiate by the angle between the two square cylinders. Although there are various methods have been proposed to control the vortex shedding have been suggested, there is still a lack of information regarding the effect of different arrangement of square cylinders in a staggered arrangement. Hopefully, the finding from this research can enhance the understanding on properties of fluid flow and sound pressure radiated over bluff bodies, hence can be used as tools to eliminate the unwanted sound and the design performance can be improved.

\section{FLOW OVER THE BLUFF BODY}

As mentioned in previous section, the flow over a bluff body is a basic knowledge and very important in order to study a flow characteristics. This kind of fundamental knowledge in fluid mechanics had been studying thoroughly in the last few decades. There are a lot of researches regarding this phenomenon. One of the interest discussions is on the flow over the bluff body in distinct form exploring this unresisting flow and noise control with distinct sizes for the bluff body. There is some interest parameter that important to evaluate such as separation, vortex shedding and wake flow. For all evaluated parameters, the square cylinder displays the smallest scale, followed by circular and ellipses, meanwhile maximum is for triangular shape [16]. This leads for the motivation in this study to look more details in the flow over the square cylinders, more specifically in the staggered arrangement.

\subsection{Flow over the Cylinder in Staggered Arrangement}

The staggered arrangement of the square cylinder is the square cylinder arranged in vertically with the spacing between cylinders to another. The study on this arrangement is important because of the fact that multiple frames of bodybuilding are placed side by side found complex flow structures and to understand the correct flow pattern for sound generation.

There is a lot of research, experimental and numerical on the flow over the cylinders in a staggered arrangement. Experimental work by D. Sumner et. al [17] found the side-by-side tandem and staggered arrangement gave flow patterns and structures of the wake for flow cases is different from the upward flow circular cylinder. This is due to the square cylinder tends to set the separation point, causing differences in critical regimes. O. Inoue et. al [18] shows that the noise produced by two square cylinders positioned side-by-side in a uniform flow at low Mach numbers affected by the distance between the cylinders. D. Chatterjee et. al [19] used method of a two-dimensional finite volume according to the Pressure Implicit with Splitting of Operators (PISO) algorithm with the fix Reynolds number 150 and for the different spacing of cylinder. Hence, by the comparison with another study also give the same result that the change of vortices wakes and sounds pressure depends on its spacing between the cylinders.

\subsection{Control of the Sound Generation on Square Cylinder}

Control to the flow mechanism all over bluff bodies is important to enhance sound losses and make it better to reduce the sound. There have two methods to control the sound generation which is an active and passive method, this study is only focused on passive wake control using a downstream flat plate. An investigation by J.Y Hwang et. al [20], for case of square cylinders with a detached flat plate found the sensitivity of the sound pressure level with the length of the plate can be collected into three regimes.

There also has another passive method to control the sound generation due to the flow pass the square cylinders with detached plate. M.F. Unal et at. [21] investigate a vortex formation from a cylinder by splitter-plate interference. From their observation, they found that the distance between the cylinder and the detached plate, $G$ gave a small interference on vortex formation for the small gap.However, for the gap rising according the critical gap, large scale vortex developed. The regimes call as prevalence and postvortex-formation. Another study by M.S.M. Ali et al. [22], the generation of noise caused by the flow had been identified for $G$ distance in range $0 \leq G \leq 7 D$ for fixed length plate $D$. From the regime, noise shrinkage is always obtained by plates, and attached plates are observed providing the highest shrinkage. In this regime, vortex interaction reinforces the elevator fluctuations on plates, and higher noise levels are generated. However, the difference in the external phase in the acoustic signal will only be observed in second regimes.

\section{NUMERICAL SIMULATION SETUP}

For the purpose to determine the effect of different arrangement of the square cylinders on the vorticity and sound propagation, simulation of the cases setup by using ANSYS Fluent software. The setup starts with the model the cases for three different arrangements, followed by solving by the software. The following sub-section explained details regarding the setup.

\subsection{Model Simulation Description}

The simulation model is shown in Figure 1. Two identical square cylinders in tandem arrangement were exposed to a free stream with constant uniform velocity. The flow was described in a Cartesian coordinate system ( $x$ and $y$ ), where inlet flow direction was $x$-axis and the $y$-axis was perpendicular to the $x$-axis. There are three cases simulated for this study which is the cases has a different arrangement of 
the second cylinder (top cylinder) in term of angle from the first cylinders. The angle used for the three cases are summarise in Table 1. The square cylinders with side length $D=10 \mathrm{~mm}$ is detached with a rigid flat plate which have a thickness of $h=0.02 D$ and its length is same with the square cylinder side $L=D$. The distance of the detached plate to the square cylinder, $G=5.5 D$ steady free-stream flow is set over the bodies at Reynolds number, $R e=150$ to 3000the Mach number, $M a=0.2$.

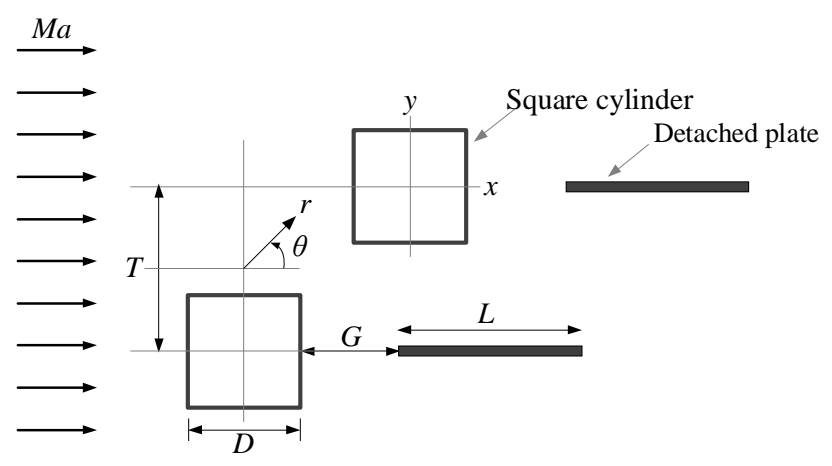

Figure 1: Schematic diagram for a square cylinder with a splitter plate(not to scale).

Table 1: Angle for different set up of cylinder \& Dimension of Boundary Condition

\begin{tabular}{cc||cc}
\hline & Angle, $\boldsymbol{\theta}$ & $\begin{array}{c}\text { Boundary } \\
\text { Condition }\end{array}$ & $\begin{array}{c}\text { Dimension } \\
(\mathbf{m m})\end{array}$ \\
\hline Case 1 & $90^{0}$ & Inlet B.C & 290 \\
Case 2 & $60^{\circ}$ & Outlet B.C & 290 \\
Case 2 & $30^{\circ}$ & Freestream B.C & 390 \\
\hline
\end{tabular}

\subsection{Governing Equation and Method of Solution}

The Large Eddy Simulation (LES) is one of the best numerical method for simulate the problem of aeroacoustics especially for the flow through the square cylinders [23]. However, in the present research, the flow past through two square cylinders in staggered arrangement was simulated by using commercial CFD code Ansys Fluent 2019 R1. The flow problem is solved by continuity and momentum equations in a given domain of interest. The equation for conservation of mass is as follow.

$\frac{\partial p}{\partial t}+\nabla \cdot(\rho \vec{v})=S_{m}$

This is the general form of equation for conservation of mass and can be used for both cases incompressible and compressible flows. The term $S_{m}$ in the equation is a source term of mass added to the continuous phase from the $2^{\text {nd }}$ phase dispersed or can be any user-defined sources. In the present study the source term, $S_{m}$ was set as zero. During the solution, the momentum equation is solved throughout the domain, and the velocity field which is the results of the solution is shared among the phases. The momentum equation is as shown in the following equation is dependent on the volume fractions of all phases through the properties $\rho$ and $\mu$. $\frac{\partial}{\partial t}(\rho \vec{v})+\nabla \cdot(\rho \vec{v} \vec{v})=\nabla p+\nabla \cdot\left[\mu\left(\nabla \vec{v}+\nabla \vec{v}^{T}\right)\right]+\rho \vec{g}+\vec{F}$

For the turbulent model, $k$-epsilon model ( 2 equations) with standard wall functions was used for the simulation. The solution is based on a pressure-based approach with Pressure-Implementation with Operator Split (PISO). Details of the parameters for the setup for turbulent modelling as summarized in Table 2.

Table 2: Detail for simulation setup

\begin{tabular}{|c|c|c|}
\hline \multicolumn{3}{|c|}{ Spatial Discretization } \\
\hline \multicolumn{3}{|c|}{$\begin{array}{l}\text { Gradient: Least Square Cell Based } \\
\text { Momentum Equation: } 2^{\text {nd }} \text { order upwind }\end{array}$} \\
\hline Solver Type & $k-\varepsilon$ Model & Solution Method \\
\hline $\begin{array}{l}\text { Pressure } \\
\text { Based }\end{array}$ & $\begin{aligned} C_{1 \varepsilon} & =1.44 \\
C_{2 \varepsilon} & =1.92 \\
C_{\mu} & =0.09 \\
\sigma_{k} & =1.0 \\
\sigma_{\varepsilon} & =1.3\end{aligned}$ & $\begin{array}{l}\text { Turbulent Dissipation Rate } \\
\left.\text { ( } 2^{\text {nd }} \text { Order Upwind }\right) \\
\text { Transient Formulation } \\
\left.\text { ( } 2^{\text {nd }} \text { Order Implicit }\right)\end{array}$ \\
\hline \multicolumn{3}{|c|}{ Starting Solution Control } \\
\hline Turbulent Kinet & $\begin{array}{r}\text { Pressu } \\
\text { Momen } \\
\text { Energy and }\end{array}$ & $\begin{array}{l}=0.3 \\
=0.7 \\
\text { bulent Dissipation Rate }=0.8\end{array}$ \\
\hline
\end{tabular}

The generations of sound due to the fluid dynamic are modelled by using a Lighthill's acoustic analogy. 2-D Green's function with the effect of a rigid boundary on sound generation is the most solution for Lighthill acoustic analogy [5]. The final equation of 2-D Curle's solution equation is as follow.

$p^{\prime}(x, y)=\frac{x_{i}}{2^{3 / 2} \pi c_{o}^{1 / 2} r^{3 / 2}} \int_{-\infty}^{\tau}\left[\frac{\partial F_{i}}{d t^{\prime}}\right] \frac{d t^{\prime}}{\sqrt{\tau-t^{\prime}}}$

\section{RESULTS AND DISCUSSION}

The results obtained from the simulation work for the three cases will be compared and discuss regarding the effect on different staggered arrangement of square cylinders detached with flat plate in term of acoustic power level, vorticity magnitude and the pressure occurred due to the flow past the square.

\subsection{Acoustics Power Level (APL)}

The acoustics power level (APL) was recorded at the same specified locations after the step then the readings were tabulated and the contour of the acoustics power level was plotted. The minimum and maximum of acoustic power level $(d B)$ for different Reynolds number and cases simulated are summarized in Table 3. From this table, the minimum APL for all condition was zero. This is due to the no sound response at the beginning of the wind flow over the cylinders. For small Reynolds number, the $A P L$ also cannot be captured by the simulation. 
Table 3: Acoustic power level $(d B)$ for three cases at different Reynolds number

\begin{tabular}{|c|c|c|c|c|c|c|}
\hline \multirow{3}{*}{ Case } & \multicolumn{2}{|c|}{$\operatorname{Re}=500$} & \multicolumn{2}{|c|}{$\operatorname{Re}=1000$} & \multicolumn{2}{|c|}{$\operatorname{Re}=1500$} \\
\hline & \multicolumn{6}{|c|}{ Acoustic Power Level $(d B)$} \\
\hline & Min & Max & Min & $\operatorname{Max}$ & Min & Max \\
\hline 1 & 0 & 0 & 0 & 1.29 & 0 & 12.5 \\
\hline 2 & 0 & 0 & 0 & 1.17 & 0 & 12.0 \\
\hline 3 & 0 & 0 & 0 & 0.87 & 0 & 9.56 \\
\hline \multirow{3}{*}{ Case } & $\operatorname{Re}$ & 2000 & $\operatorname{Re}$ & 500 & $\mathrm{Re}$ & 3000 \\
\hline & \multicolumn{6}{|c|}{ Acoustic Power Level $(d B)$} \\
\hline & Min & $\operatorname{Max}$ & Min & $\operatorname{Max}$ & Min & $\operatorname{Max}$ \\
\hline 1 & 0 & 18.7 & 0 & 24.6 & 0 & 30.2 \\
\hline 2 & 0 & 18.6 & 0 & 24.1 & 0 & 30.3 \\
\hline 3 & 0 & 15.2 & 0 & 21.8 & 0 & 27.4 \\
\hline
\end{tabular}

There are other types of simulations which are more suitable or have a better ability in capturing the $A P L$ of the flow that had been studied by other researchers. Direct numerical simulation $(D N S)$ is the one of simulation which has the better ability in capturing acoustics sound level, but the requirements are so large that this method is not a practical approach for a simple case like two dimensional two square cylinders with detaching flat plate. Besides that, there is another direct method which is the large eddy simulation $(L E S)$ that can be used to study acoustics sound level. This is the cheaper method compared to the DNS and it allows for more complex flow configuration at higher Reynolds numbers to be resolved but it is still computationally demanding.

For the highest Reynolds number simulated, $R e=3000$, the $A P L$ were captured. The contour of the APL for three cases was shown in Figure 2.Figure 2(a) shows the contour of the APL which is the cylinder detaches with flat plate in $90^{\circ}$ position. From the results, the maximum $A P L$ captured is 30.0 $d B$. It is occurred at the shear layer of upstream body of top cylinder and downstream body of the bottom cylinder. In position of $60^{\circ}$ for case 2 showed in Figure 2(b), it had created small wave at the top cylinder, where thehighest $A P L$ is formed at upstream of cylinder.Figure 2(c) for the results of case 3, the maximum $A P L$ was captured in the same structure for upstream and downstream body of the cylinder from the top cylinder.

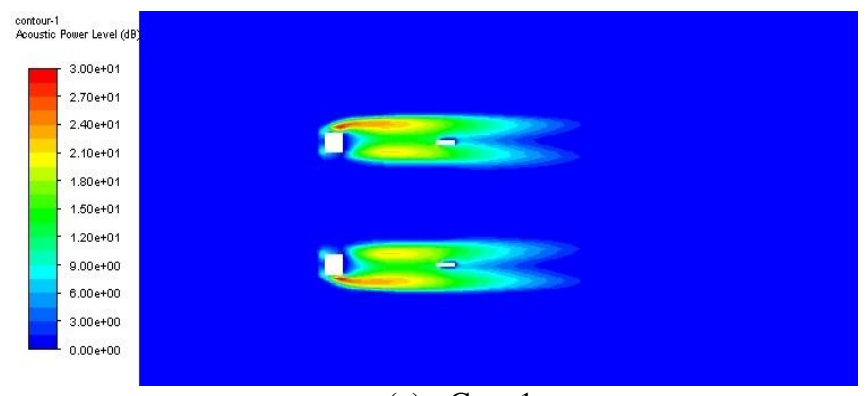

(a) Case 1

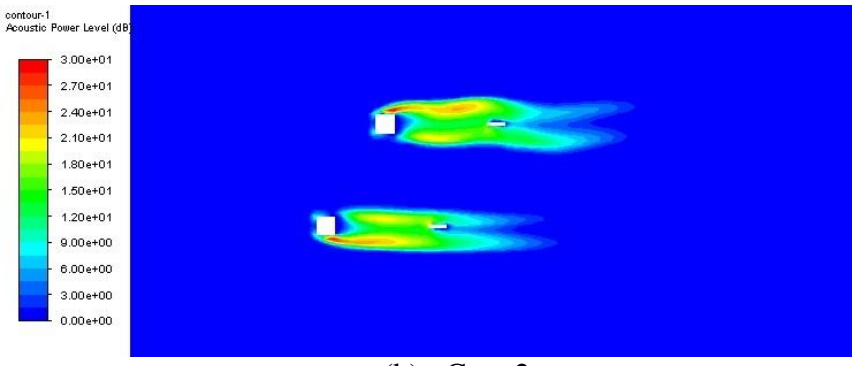

(b) Case 2

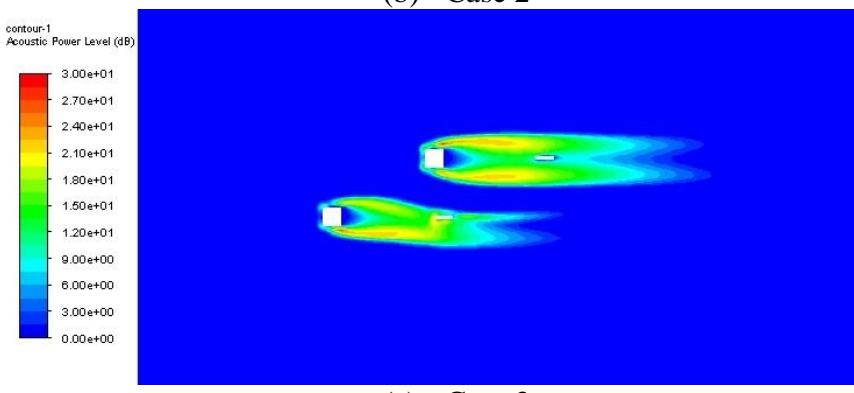

(c) Case 3

Figure 2: Contour of acoustic power level for $R e=3000$

\subsection{Vorticity Magnitude}

The vortex shedding is visualized throughout the contours and vectors of velocity magnitude. The value of velocity magnitude was increased with the increase of Reynolds number as shown in Table 4.An arrangement of the square cylinders also gave a significant in vorticity magnitude where it was decreased from case 1 to case 3.

Table 4: Vorticity magnitude for three cases at different Reynolds number

\begin{tabular}{|c|c|c|c|c|c|c|}
\hline \multirow{3}{*}{ Case } & \multicolumn{2}{|c|}{$\operatorname{Re}=500$} & \multicolumn{2}{|c|}{$\operatorname{Re}=1000$} & \multicolumn{2}{|c|}{$\mathrm{Re}=1500$} \\
\hline & \multicolumn{6}{|c|}{ Vorticity Magnitude } \\
\hline & Min & $\operatorname{Max}$ & Min & Max & Min & Max \\
\hline \multirow{2}{*}{1} & 1.34 & 8.84 & 1.27 & 1.71 & 1.82 & 2.50 \\
\hline & $e-03$ & $\mathrm{e}+02$ & e-03 & $e+03$ & $e-02$ & $e+03$ \\
\hline \multirow{2}{*}{2} & 3.45 & 8.83 & 9.26 & 1.68 & 0.43 & 2.59 \\
\hline & e-03 & $\mathrm{e}+02$ & e-03 & e+03 & $1 e-2$ & e+03 \\
\hline \multirow{2}{*}{3} & 0.82 & 8.83 & 9.35 & 1.73 & 1.96 & 2.36 \\
\hline & e-03 & $\mathrm{e}+02$ & e-03 & $\mathrm{e}+03$ & $\mathrm{e}-02$ & $\mathrm{e}+03$ \\
\hline \multirow{3}{*}{ Case } & $\mathrm{Re}=$ & 000 & $\mathrm{Re}=$ & 500 & $\operatorname{Re}=$ & 3000 \\
\hline & \multicolumn{6}{|c|}{ Vorticity Magnitude } \\
\hline & Min & Max & Min & Max & Min & Max \\
\hline \multirow{2}{*}{1} & 1.60 & 2.56 & 2.09 & 2.66 & 2.23 & 3.17 \\
\hline & e-02 & $\mathrm{e}+03$ & e-02 & $e+03$ & e-02 & e+03 \\
\hline \multirow{2}{*}{2} & 1.99 & 2.56 & 3.98 & 2.55 & 3.62 & 3.04 \\
\hline & $\mathrm{e}-02$ & $\mathrm{e}+03$ & $\mathrm{e}-02$ & $e+03$ & $e-02$ & $e+03$ \\
\hline \multirow{2}{*}{3} & 1.48 & 2.93 & 3.01 & 2.23 & 3.64 & 2.67 \\
\hline & e-02 & $e+03$ & $\mathrm{e}-02$ & $e+03$ & $e-02$ & $e+03$ \\
\hline
\end{tabular}

For the vorticity contour, again the comparison was made for higher Reynolds number simulated, at $R e=3000$. The results showin Figure 3 clearly shows that the vortex shedding occurred downstream of the cylinders. From the figure, the vortices shedding behind the downstream cylinders shows do not have a quite significant on the different arrangement of 
cylinders, especially for case 1 . It is because of the weak interactions and due to relatively big streamwise gap between the upstream and downstream of the cylinders. For case 3, a wide wake was observed behind the cylinders and a narrow wake observed behind the second cylinders. The vortices generating from the downstream of second cylinder fluctuated and switches the direction (up and down), and this affect normally controlled by the wakes generated behind the downstream of first cylinder.

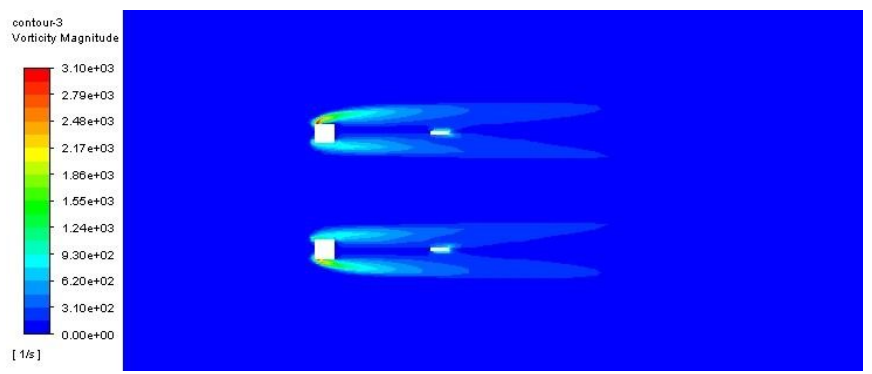

(a) Case 1

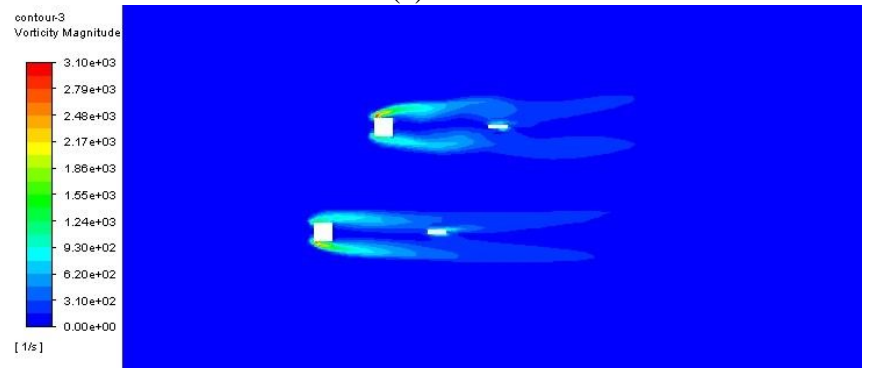

(b) Case 2

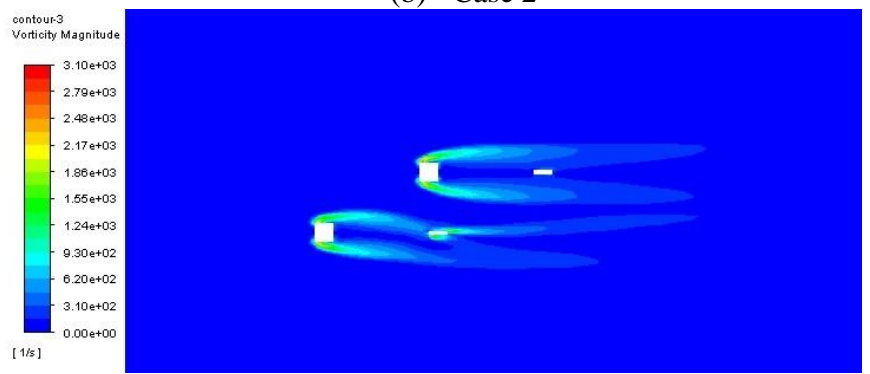

(c) Case 3

Figure 3: Contour of vorticity magnitude for $R e=3000$

\subsection{Dynamic Pressure}

Dynamic pressure represented the kinetic energy of a moving fluid. As the kinetic energy also gave a significant on the vorticity and sound propagation due to the different arrangement of square cylinders, the behaviour of the kinetic energy were discuss in this section. Table 5 shows the minimum and maximum of dynamic pressure $(\mathrm{Pa})$ for different Reynolds number. From the table, the kinetic energy was increase with the increasing of the Reynolds number. In term of arrangement, the case 1 gave the higher kinetic energy compared to other cases.
Table 5: Dynamic Pressure for three cases at different Reynolds number

\begin{tabular}{|c|c|c|c|c|c|c|}
\hline \multirow{3}{*}{ Case } & \multicolumn{2}{|c|}{$\mathrm{Re}=500$} & \multicolumn{2}{|c|}{$\operatorname{Re}=1000$} & \multicolumn{2}{|c|}{$\operatorname{Re}=1500$} \\
\hline & \multicolumn{6}{|c|}{ Dynamic Pressure $(\mathrm{Pa})$} \\
\hline & Min & Max & Min & Max & Min & Max \\
\hline \multirow[t]{2}{*}{1} & 1.85 & 5.89 & 3.73 & 2.31 & 9.94 & 5.20 \\
\hline & e-04 & e-01 & $e-04$ & $e-00$ & e-04 & $e+00$ \\
\hline \multirow[t]{2}{*}{2} & 0.23 & 5.86 & 1.04 & 2.30 & 2.90 & 5.16 \\
\hline & e-04 & e-01 & e-04 & $e+00$ & e -04 & $\mathrm{e}+00$ \\
\hline \multirow[t]{3}{*}{3} & 1.10 & 5.63 & 2.72 & 2.26 & 5.22 & 4.06 \\
\hline & e-04 & $\mathrm{e}-01$ & $\mathrm{e}-04$ & $\mathrm{e}+00$ & e-04 & $\mathrm{e}+00$ \\
\hline & \multicolumn{2}{|c|}{$\operatorname{Re}=2000$} & \multicolumn{2}{|c|}{$\operatorname{Re}=2500$} & \multicolumn{2}{|c|}{$\operatorname{Re}=3000$} \\
\hline \multirow[t]{2}{*}{ Case } & \multicolumn{6}{|c|}{ Dynamic Pressure $(\mathrm{Pa})$} \\
\hline & Min & $\operatorname{Max}$ & Min & Max & Min & $\operatorname{Max}$ \\
\hline \multirow[t]{2}{*}{1} & 3.78 & 8.90 & 8.74 & 1.40 & 2.18 & 2.04 \\
\hline & e-04 & $\mathrm{e}+00$ & e-04 & $\mathrm{e}+01$ & e-03 & $\mathrm{e}+01$ \\
\hline \multirow[t]{2}{*}{2} & 6.09 & 8.60 & 1.38 & 1.34 & 3.22 & 1.93 \\
\hline & e-04 & $\mathrm{e}+00$ & $\mathrm{e}-03$ & $\mathrm{e}+01$ & $\mathrm{e}-03$ & $e+01$ \\
\hline \multirow[t]{2}{*}{3} & 2.08 & 9.10 & 2.49 & 1.44 & 3.25 & 2.09 \\
\hline & e-03 & $e+00$ & e-03 & $e+01$ & e-03 & $\mathrm{e}+01$ \\
\hline
\end{tabular}

Observation for the contour of dynamic pressure at Reynolds number, $R e=3000$ were shown in Figure 4. The maximum dynamic pressure for case 1 occurred at lower stream of top cylinder and upper stream at bottom cylinder. Moreover, the dynamic pressure distribution for the case 1 is symmetry which is shows that the dynamic pressure was distributed evenly. For arrangement of the cylinders as case 2 and 3, the maximum pressure is higher for the top cylinder. The high pressure on the top of the square cylinder gives the effect of bottom cylinder. In figure it can see that the minimum pressure at area bottom cylinder almost suppurate from the flat plate that detached with cylinder.

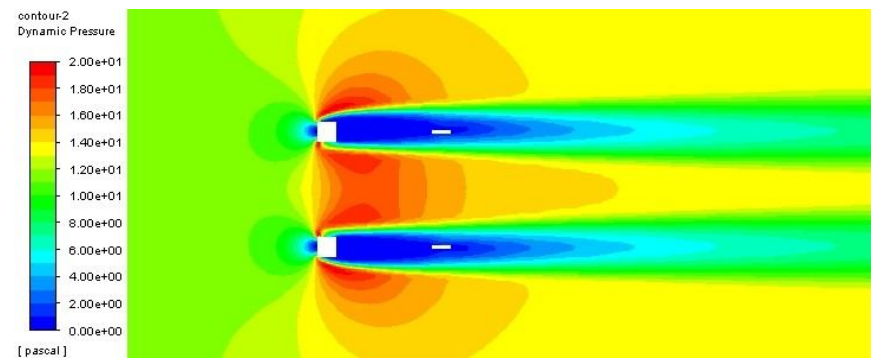

(a) Case 1

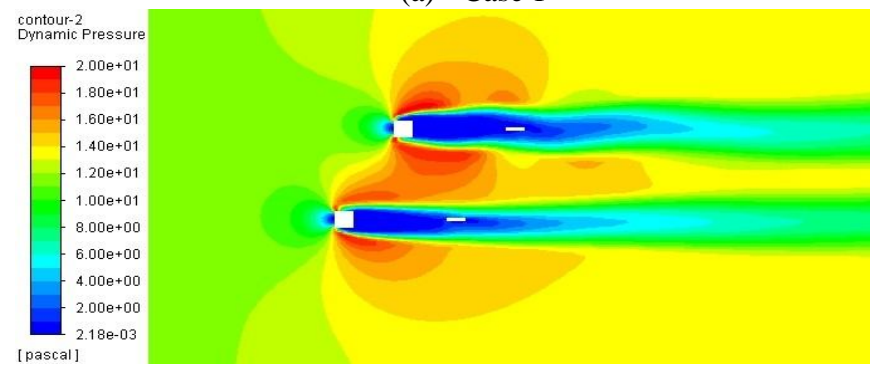

(b) Case 2 


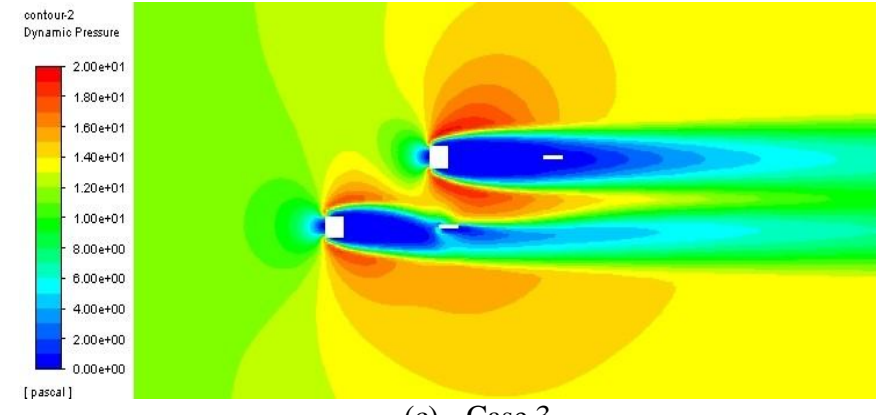

(c) Case 3

Figure 4: Contour of dynamic pressure for $R e=3000$

\subsection{Total Pressure}

The total pressure distribution for the rest of the Reynolds number simulated was presented in Figure 5. From the figure, figure the increasing of the Reynolds number was given the effect of total pressure in each case. The pressure distribution clearly shows that the higher pressure generated by the higher Reynolds number. In term of effect of arrangement, it do not show a big significant for the pressure far from the cylinder.

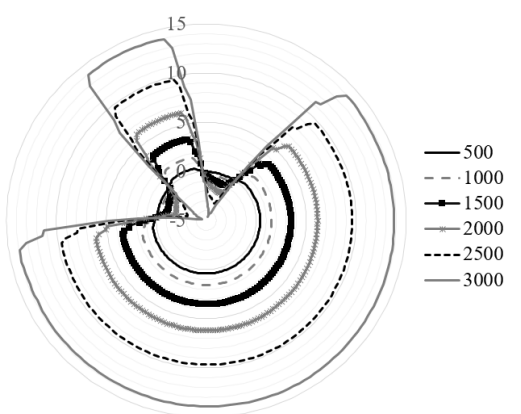

(a)

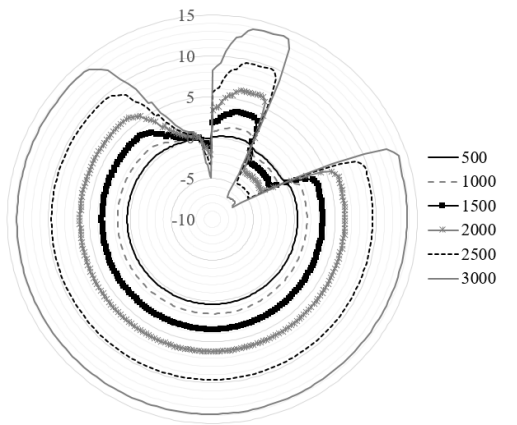

(b)

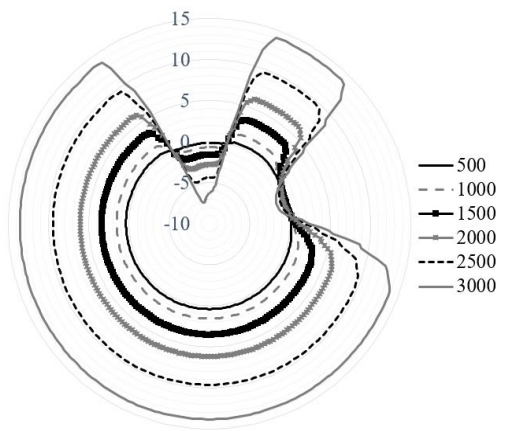

(c)

Figure 5: Directivity of total pressure

\section{CONCLUSION}

The effect of staggered arrangement of square cylinders with detached flat plat on vorticity and sound generation has been investigated. The effect of the three cases of arrangement with different angle, $\theta=90^{\circ}, 60^{\circ}$ and $30^{\circ}$ on vorticity and sound pressure propagation was investigated in detail and compared with the single cylinder (from literature). It was found that the directivities and magnitudes of the sound depend strongly on the angle of arrangement near the cylinder while the effect not has a quite significant at far field. The vortices are shed from the downstream cylinder only and the amplitudes of the vortex shedding for two cylinders are smaller than the single cylinder case. The magnitude of the sound is also smaller than the single cylinder case. For the magnitude of the dynamic and sound pressure, the results shows that both magnitudes is larger for the case of two square cylinder compare to single cylinder case. For the visualization of acoustic pressure level by using the present method of simulation, the acoustic pressure only is captured at the higher Reynolds number. These due to the sound pressure are too small compare to the ambient pressure and there are needs for another method such as DNS or LES.

\section{ACKNOWLEDGMENT}

Authors acknowledge the financial support to Research Fund E15501, Research Management Centre, UniversitiTun Hussein Onn Malaysia (UTHM).

\section{REFERENCES}

1. B. Manshoor, I. Zaman, I, A. Khalid, M.F. Ghazali and M.K. Khandelwal. Effect of streamwise spacing on the sound generated by flow through two square cylinders in tandem arrangement. International Journal of Advanced Trends in Computer Science and Engineering. vol.9, No.1.1, pp. 534-521, 2020.

2. I. Zaman, M. Mohamed Salleh, B. Manshoor, A. Khalid and S. Araby S. The application of multiple vibration neutralizers for vibration control in aircraft. Applied Mechanics and Materials, 629, pp.191-196 2014

3. Wang X., Bushan S., Manshoor B., Luke E., Sescu A., Hattori Y., Thompson D., and Walters K. Dynamic hybrid RANS/LES assessment of sound generation and propagation from flow over a circular cylinder., In 2018 AIAA/CEAS Aeroacoustics Conference, AIAA AVIATION Forum, (AIAA 2018-3592). 2018 https://doi.org/10.2514/6.2018-3592.c1

4. M.S.M. Ali, Aeolian Tones Radiated from Flow Over Bluff Bodies. Open Mech. Eng. J., vol. 7, no. 1, pp. 48-57, 2013 https://doi.org/10.2174/1874155X01307010048

5. A. Zakaria and M. S. N. Ibrahim, Experimental evaluation of multiple savonius turbines in oblique and cluster configurations. International Journal of Emerging Trends in Engineering Research., vol. 7, no. 12, pp. 790-793, 2019 https://doi.org/10.30534/ijeter/2019/10712201 
6. N. Curle. The influence of solid boundaries upon aerodynamic sound. Proc $R$ Soc A., vol. 231, pp. 505-514, 1955

7. L. Du and X. Sun. Suppression of vortex-induced vibration using the rotary oscillation of a cylinder. Phys Fluids 27:023603, 2015 https://doi.org/10.1063/1.4913353

8. X. Huang. Feedback control of vortex shedding from a circular cylinder. Exp Fluids., vol. 20, pp. 218-224, 1996

9. S.J. Illingworth, H. Naito, K. Fukagata. Active control of vortex shedding: an explanation of the gain window. Phys Rev E., vol. 90:043014, 2014

10. M.M. Zhang, Y. Zhou andL. Cheng. Closed loop manipulated wake of a stationary square cylinder. Exp Fluids., vol. 39, pp. 75-85, 2005 https://doi.org/10.1007/s00348-005-0979-3

11. Y.G. Liu and L.H. Feng. Suppression of lift fluctuations on a circular cylinder by inducing the symmetric vortex shedding mode. Journal of Fluid Structure., vol. 54, pp. 115-122, 2015

12. G. Nati G, M. Kotsonis, S. Ghaemi and F. Scarano. Control of vortex shedding from a blunt trailing edge using plasma actuators. Exp.Thermal Fluid Sci.,vol. 46, pp. 199-210, 2013

13. J.J. Wang, K.S. Choi, L.H. Feng, T.N. Jukes and R.D. Whalley.Recent developments in DBD plasma flow control. Prog Aerospace Science., vol. 62, pp. 52-78 2013

14. A. Roshko A.On the drag and shedding frequency of two-dimensional bluff bodies. Technical Report 3169, National AdvisoryCommittee for Aeronautics 1954

15. C.J. Apelt, G.S. West and A.A. Szewczyk. The effects of wake splitter plates on the flow past circular cylinder in the range104 $<\boldsymbol{R}<5 \times \mathbf{1 0 4}$. J Fluid Mech., vol. 61, pp. $187-198,1973$ https://doi.org/10.1017/S0022112073000649

16. B. Manshoor and A. Khalid. Numerical investigation of the circle grids fractal flow conditioner for orifice plate flow meters.Applied Mechanics and Materials 229-231, pp. 700-704, 2000.

17. D. Sumner, S.S.T. Wong, S.J. Price and M. P. Païdoussis, Fluid behaviour of side-by-side circular cylinders in steady cross-flow,J. Fluids Struct., vol. 13, no. 3, pp. 309-338, 1999.
18. O. Inoue, W. Iwakami, N. Hatakeyama, O. Inoue, W. Iwakami, and N. Hatakeyama, Aeolian tones radiated from flow past two square cylinders in a side-by-side arrangement.Physics of Fluids., vol. 18, no. 4, 2006 https://doi.org/10.1063/1.2191847

19. D. Chatterjee, G. Biswas, and S. Amiroudine, Numerical simulation of flow past row of square cylinders for various separation ratios.,Comput. Fluids, vol. 39, no. 1, pp. 49-59, 2010

20. J.Y. Hwang, K.S. Yang and S. H. Sun. Reduction of flow-induced forces on a circular cylinder using a detached splitter plate.,Phys. Fluids, vol. 15, no. 8, pp. 2433-2436, 2003 https://doi.org/10.1063/1.1583733

21. M.F. Unal and D.Rockwell. On vortex formation from a cylinder: Part2. Control by splitter plate interference.,J. Fluid Mech., vol. 190, no. 9, pp. 513-529, 1988.

22. M.S.M. Ali, C.J. Doolan and V. Wheatley.Aeolian Tones Generated by a Square Cylinder with a Detached Flat Plate.,AIAA J., vol. 51, no. 2, pp. 291-301, 2013 https://doi.org/10.2514/1.J051378

23. K.K. Nirmal. Numerical studies on supersonic fluid flow past a cavity involving pressure field and aeroacoustic effects using LES approach. International Journal of Emerging Technologies in Engineering Research., vol. 6, no. 1, pp. 65-70, 2018 